\title{
Non-Invasive Monitoring of Blood Glucose Concentration Based on Insulin Secretion Level Using NIR Spectroscopy for Diabetes Detection
}

\author{
D. Arul Kumar' ${ }^{1}$, T. Jayanthy ${ }^{2}$ \\ ${ }^{1}$ Research Scholar, Department of Electronics and Communication Engineering, Sathyabama Institute of Science \\ and Technology, Chennai, India, ${ }^{2}$ Principal, Panimalar Institute of Technology, Chennai, India
}

\begin{abstract}
Diabetes claims millions of lives every year. Diabetes Mellitus is a metabolic disorder that occurs due to the increase in blood sugar level. The blood sugar level control by insulin secreted from pancreas. The diabetes classify as type 1 and type 2 depending on insulin secretion. The type 2 diabetes is more common in people than type 1 diabetes. Traditionally, the blood glucose level estimate with glucometer and blood test. However, the test require invasive blood pricking for blood glucose measurement. The measured blood glucose value also varies due to amount of blood taken for testing, part of blood used for testing, depth of finger pricking, physical activity, stress and underlying illness. Hence, the insulin secretion from pancreas monitor for accurate prediction of blood glucose level. The blood glucose level varies with respect to insulin secretion from pancreas. In this paper, we propose a non-invasive approach to estimate blood glucose accurately through NIR spectroscopy for diabetes control. The NIR spectroscopy signal obtained from pancreas process through Rational Dilation Wavelet Transformto determine the insulin secretion level. The non-invasive NIR spectroscopy method measures glucose level with $90 \%$ accuracy compared to lab results.
\end{abstract}

Keywords: Type 2 Diabetes, RADWT, NIR spectroscopy, Insulin.

\section{Introduction}

Diabetes mellitus characterizes by the abnormal increase of sugar level in the blood. When the glucose level in the blood increases, it induces the insulin release from the pancreas. Insulin causes fat cells and muscle to remove glucose from the blood and causes the liver to metabolize glucose and thus reduceblood sugar to normal levels. People with diabetes have high levels of blood sugarsince, pancreas does not produce insulin. The diabetic person have minimal count of $\beta$-cells in pancreas which secretes low insulin.

The most general forms of diabetes are type 1,type 2 diabetes, and gestational diabetes. The type 1 diabetes is an autoimmune disorderwhere, the body attacks the pancreas making it unable to produce insulin resulting in minimal insulin secretion. Thetype 2 diabetes cause due to factors such as metabolic disorders, age, overweight, obesity, physical inactivity and unhealthy food consumption. The $\beta$ cell in pancreas produce insufficient insulin coupled with organ resistance to insulin and result in high blood glucose level. The gestational diabetes occurs during pregnancy.

Currently, the blood glucose level control by medication and insulin injection coupled with diet control and physical activity. The blood glucose level determine by blood test or glucometer. The first introduced blood glucose meter was Ames Reflectance Meter. The Ames Reflectance Meter enables diabetic patients to monitor the blood glucose levels themselves. Reflomat is another portable and compact blood glucose meter designed for health care professionals. The Reflomat requires only a small amount of blood to determine blood glucose level. The foresaid meter needs extraction of blood samples through fingertip pricking, ear pricking. The blood glucose monitoring require taking test for up to 4 times every day. Therefore, continuously pricking of finger, ear for blood to determine blood glucose level may cause infection, and calcified nodules. The blood sugar level estimated by glucometer are inaccurate due to damaged blood cells. Further, the frequent skin pricking 
is uncomfortable, inconvenient and cause skin irritation. Hence, precise, convenient, safe and comfortable blood glucose measurement method is required.

The blood glucose also measure by non-invasive method. The polarization change is the first introduced non-invasive technique where combination of helium and neon based laser light is coupled to a linear polarizer and the phase matches with the rotation vector is relative to glucose.

Raman Spectroscopy can detect low frequency modes. A Surface Enhanced Raman Spectroscopy (SERS) detects the interaction between glucose molecules and SERS active surface. The monochromatic light on molecule result in photon energy shift. The energy shift of photon is proportional to molecular bond vibration. The molecular vibration have specific Raman spectrum band. The spectrum band featuresvary for different molecules such as glucose.

Fluorescent spectroscopy is an electromagnetic spectroscopy technique that can analyze a sample's fluorescence. Fluorescent Spectroscopy utilizes visible light spectrum, to detect energy emitted at different wavelength from glucose molecule. Near-Infrared (NIR) spectroscopy employs near-infrared light of wavelength $780-2500 \mathrm{~nm}$. In NIR spectroscopy, glucose level estimates with respect to the variations in the light intensity. Mid-Infrared (MIR) spectroscopy is developed from NIR spectroscopy that utilizes the near infrared light of wavelength 2500-10000nm. MIR spectroscopy is sharper and does not penetrate through the skin effectively. Absorption spectroscopy in the infrared (IR) region is a significant technique for finding unidentified biological substances in aqueous solutions. The technique is based on the process that every molecule has particular resonant absorption peaks. These peaks are caused by molecule's rotational and vibrational oscillations.

Literature Survey: Non-invasive blood glucose monitoring perform through in vitro and in vivo analysis of Differential Continuous Wave Photo Acoustic Spectroscopy (DCW-PAS). The DCW-PAS approach uses amplitude modulation withdual wavelengths of light to identify glucose concentration level. The analysis compares DCW-PAS evaluations including results from invasive blood glucose sensor evaluations of healthy people's Oral Glucose Tolerance Tests (OGTTs). The blood glucose level estimation from photo-acoustic signal and invasive sensors have good correlation. ${ }^{[1]}$

The blood glucose level measure with plasmonic sensor made of barium flint glass, gold film andsilicon nitride $\left(\mathrm{Si}_{3} \mathrm{~N}_{4}\right)$ substrate. The plasmonic sensor rejects infrared wavelength on normal incidence because of coupling between plasmonic wave and incident plane wave. The impact of glucose concentration or ambient refraction analyze for plasmonic structure sensitivity toblood glucose. ${ }^{[2]}$

A Spoof Surface Plasmon Polariton (SSPP) endfire sensor monitors aqueous glucose solutions and measures on-body glucose. The SSPP endfire sensor radiates an endfire beam into a glucose water solution with minimal effective aperture. At the sensor's CPW port, a set of triangular ground planes suppresses the side-lobes and limit glucose sensing. The SSPP endfire sensor's slow wave nature provides the way for measuring glucose concentrations with enhanced sensitivity. ${ }^{[3]}$

Non-invasive blood glucose monitoring offers an effective solution to diagnose patients with diabetes the glucose response characterize at low Radio Frequency (RF) signals. The relative permittivity and conductivity of aqueous solutions obtain for various glucose concentrations using an impedance analyzer in the frequency range $1 \mathrm{KHz}$ to $1 \mathrm{MHz}$. Further, the blood impedance measure for glucose monitoring the bio-impedance, measure from forearm during cardiac cycle with polygraph at $1000 \mathrm{~Hz}$. The difference in bio impedance evaluate for cardiac cycle. The influence of blood glucose concentration on bio impedance evaluate to remove tissue influence on blood glucose concentration. ${ }^{[4]}$

The design of a microwave sensor for non-invasive monitoring of blood glucose concentration is presented. Three distinct microwave resonator structures analyze as suitable candidates. The microwave resonator has an open structure to place patient's finger. The finger's shape and size should fit in the resonator. The variation in blood glucose concentration alters the tissue's dielectric properties and changes the structure's resonant frequency. ${ }^{[5]}$

A combined millimeter-wave radar system detects various glucose concentration levels of duplicate blood samples made in the laboratory. The mm-wave radarnoninvasively monitors blood glucose of patients with diabetes. The mm-wave radar signal with Discrete Time 
Fourier Transform finds various glucose concentrations in hemoglobin samples. ${ }^{[6]}$

The near infrared spectroscopy non-invasively measures glucose concentration in blood. The infrared light pass through finger and blood glucose concentration evaluate by calculating absorbance through BeerLambert law. The infrared absorbance is equivalent to blood glucose concentration and finger thickness. ${ }^{[7]}$

A sensitive Glucose fringe field Microstrip Line (MLIN), Material under Test (MUT) - glucose as substrate detects concentration of glucose and ports. The electromagnetic field from MLIN interacts with MUT and show variation in $\left|S_{11}\right|_{\text {min }}$. The glucose concentration estimate by Single variate, multivariate and multivariate estimation with bin correlation algorithm. ${ }^{[8]}$

An implanted sensor with telemetry system monitors subcutaneous tissue glucose for long term in diabetic patients. The implantable sensor consist of immobilized glucose oxidase membrane, polydimethylsiloxanemembrane, catalase connected to electrochemical oxygen detection and telemetry system for wireless data transmission. ${ }^{[9]}$

A planar microwave sensor monitors glucose level continuously. The sensor element contains four different hexagonal-shaped complementary split ring resonators (CSRR) resembling honey-cell pattern. The resonator was fabricated on a FR4 dielectric substrate and connected to planar micro strip with dielectric substrate. The CSRRs were connected through microstrip transmission line to a radar system operating at 2.4-2.5 GHz. The combined sensor system achieves a good sensitivity in detecting the glucose levels that dissolves in the blood similar to aqueous solutions. ${ }^{[1} \mathrm{A}$ substrate integrated waveguide (SIW) planar sensor's design measured blood glucose concentration non-invasively. The SIW planar sensor's structure resembles traditional band stop filter. The SIW planar sensor, yields a considerable and localized field improvement in the sensing region, the inter-digital arms and the slots on the SIW planar sensor cavity's upper conductor are utilized. Additionally, fingertip is utilized as material under test (MUT) and the effects of finger prints and the finger's displacement are analyzed. ${ }^{[11]}$

The $\mathrm{ZnO}$ based ultrasonic piezoelectric MicroElectronics Mechanical Systems (MEMS) receiver monitors blood glucose. The radial displacement and the surface of the ultrasonic piezoelectric MEMS receiver produce voltage due to pressure and stress. The voltage vary with respect to blood concentration level. The simulation analysis of glucose data showed agreeable correlation with glucometer reading. ${ }^{[12]}$

The blood glucose monitor with RF/microwave technology. The RF sensor measure blood glucose level by detecting dielectric changes of blood. The dielectric variation due to glucose causes the sensor frequency to shift below $8 \mathrm{Mhz}$. The frequency shift also occur due to blood layer, skin, fat, pressure and position of finger. ${ }^{[13]}$

A wearable, minimum invasive autonomous and pseudo-continuous blood glucose monitoring. This wearable micro system design obtains a whole blood sample from a little lanced skin wound utilizing a new micro-actuator based on a shape memory alloy (SMA) and straightly measures the blood glucose level from the blood sample. ${ }^{[14]}$

A wirelessly powered implantable electrochemical sensor system monitors blood glucosecontinuously. The system was powerby $13.56 \mathrm{MHz}$ inductive link and an ISO 15693 radio frequency identification (RFID) standard for telemetric communication. The sensor system comprises awinding ferrite antenna, a RFID front-end, a 10-bit sigma-delta analog to digital converter (ADC), a long-term glucose sensor, an onchiptemperature sensor, a potentiostatand a digital baseband for controlling and processing protocol. A high frequency (HF) external readerpowers, commands and configures the sensor system directly. The off-chip support circuitry requiresa glucose micro-sensor and a tuned antenna. ${ }^{[15]}$

\section{Methodology}

The non-invasive technique of diabetes detection is performed by measuring the blood glucose concentration through NIR spectroscopy. The NIR spectroscopy measures blood glucose concentration by evaluating insulin secretion from pancreas. The NIR sensor is placed on the pancreas region.Figure 1 shows the overview of diabetes prediction withreflected infrared from pancreas.

Pancreas is a gland that is six inches long and located in the abdomen. It is flat and pear shaped, surrounded by liver, small intestine, spleen, stomach. The pancreas's endocrine cells produce hormones. Hormones are substances that regulate or control particular functions in the body. Hormones are generally formed in one part of the body and passed through the blood to react on another part of the body. The two important pancreatic 
hormones are insulin and glucagon. The endocrine cells such as the islet cells present in the pancreas produce and secrete glucagon and insulin in the blood. Glucagon increases the blood sugar level while insulin lowers the blood sugar level. The two hormones function together to maintain proper blood sugar level. The figure 2 shows the structure of pancreas.

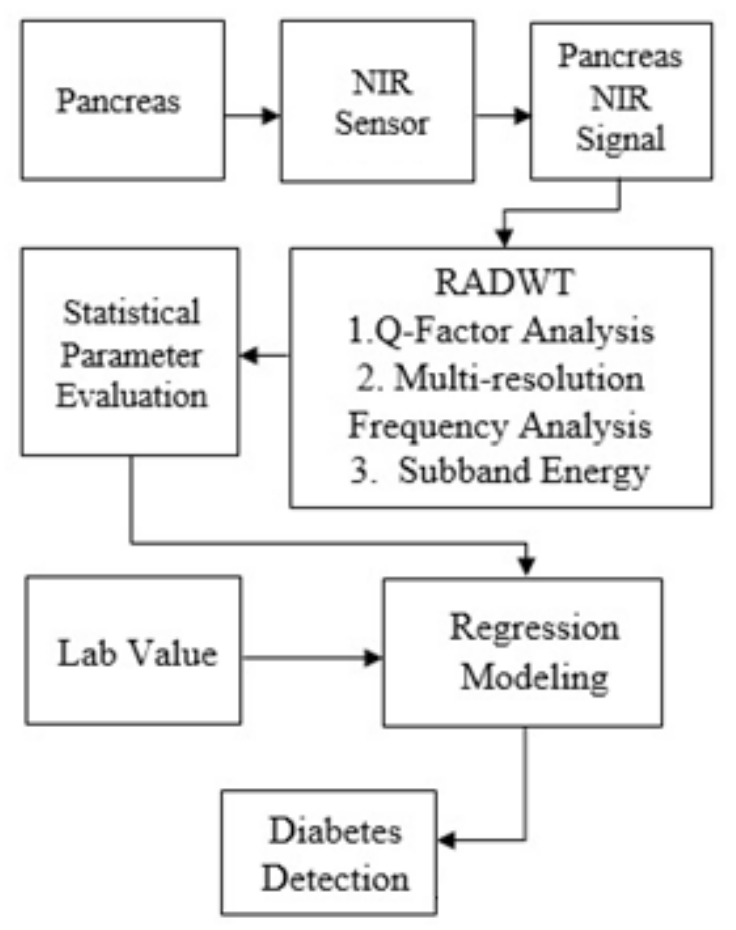

Figure 1. Overview of diabetes detection with NIR through RADWT algorithm.

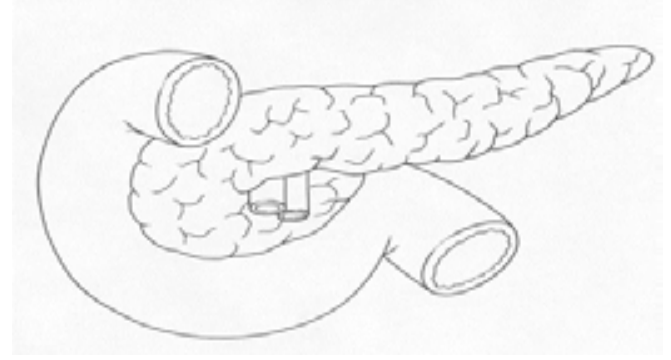

Figure 2. Structure of Pancreas

To find the insulin secretion level from pancreas, NIR spectroscopy is used. NIR spectroscopy is a spectroscopic technique that utilizes the electromagnetic spectrum's from near infrared region. NIR spectroscopy is used in various medical applications such as pulse oximetry, blood sugar level monitoring, neurology and urology. The NIR sensor is placed on the pancreas region as shown in figure 3 . The reflected infrared signal from pancreas acquire with data acquisition tool. The infrared signal from pancreas of normal and diabetic person. The pancreas infrared signal obtain for before meal and after meal condition. The acquired infrared signalsprocesses through Rational Dilation Wavelet Transform (RADWT) to determine infrared variation due toinsulin secretion. In RADWT, the Q-factor value change for resolution and subband energy level of pancreas NIR signal.

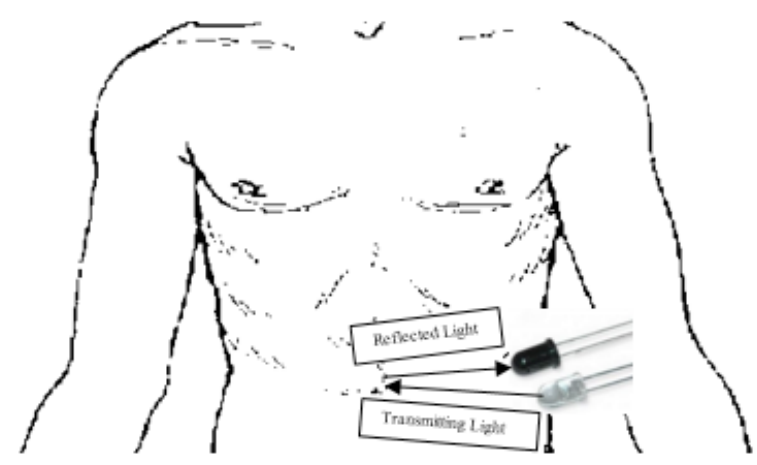

Figure 3. NIR Diode Placed on the Pancreas Region

Rational Dilation Wavelet Transform (RADWT): The Dyadic Wavelet Transform is an effective transformation tool for sparsely representing the smooth signals and it is a constant Q-factor transform and a critically sampled wavelet transform. However, its frequency resolution is poor and has a low Q-factor. The Dyadic Wavelet Transform is not effective for processing NIR signals with oscillatory nature. Some techniques like Cosine Modulated Filter Banks, Short Time Fourier Transform (STFT), wavelet packets are traditionally used for oscillatory type signals rather than using dyadic wavelet transform. The transforms do not have a constant Q-factor. So an efficient transform with constant and high Q-factor and high frequency resolution is required. Another category of wavelet transforms like overcomplete wavelet transform performs efficiently than critically sampled wavelet transforms like dyadic wavelet transform. Overcomplete wavelet transforms extend an $N$-point NIR signal to a set of $M$ coefficients with $M>N$. Several overcomplete invertible wavelet transforms such as double density wavelet transform, dual tree complex wavelet transform and undecimated wavelet transform exists. These wavelet transforms achieve over completeness through increasing only the temporal sampling in all frequency bands. The frequency spacing between adjacent frequency bands should be reduced to utilize the overcomplete wavelet transform's redundancy. These overcomplete wavelet transforms are based on rational dilations. The figure 4 represents the Rational Dilation Wavelet Transform (RADWT) where 
$H(\omega)$ is high pass filter, $G(\omega)$ is low pass filter, $p$ and $q$ are rational dilation factors and $s$ is redundancy factor.

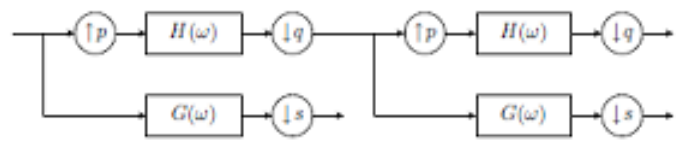

\section{Figure 4. RADWT}

The selection of dilation factor close to one, a wavelet can be dilated from scale to scale and multi resolution frequency analysis of the NIR signal can be performed. Additionally, the dilation factors $\mathrm{p}$ and $\mathrm{q}$ and the redundancy factor s enhance the Q-factor and frequency resolution of the NIR signal. The Q factor of the signal resolution depend on the rational dilation factors $q$ and $p$. The filter banks of RADWT is shown in figure 5 .

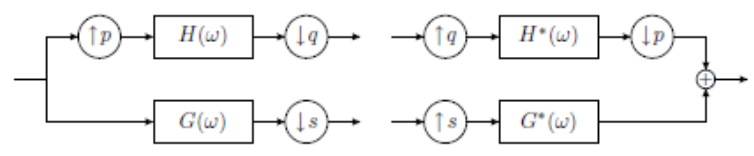

Figure 5. Filter Bank of the proposed RADWT

The perfect reconstruction filters for the filter bank shown in figure 5 is derived. The parameters $\mathrm{p}$, $\mathrm{q}$ and $\mathrm{s}$ are positive integers that satisfy $1 \leq \mathrm{p}<\mathrm{q}$ and $\mathrm{p} / \mathrm{q}$ $+1 / s \geq 1$. The integers $p, q$ are coprime. For perfect reconstruction $p+1=q=s$. The only condition for perfect reconstruction filters is

$$
\frac{1}{p q}\left|H\left(\frac{\omega}{p}\right)\right|^{2}+\frac{1}{s}|G(\omega)|^{2}=1 \text { for } \omega \in[0, \pi]
$$

Finally, the perfect reconstruction filters are given as

$$
\begin{aligned}
& H(\omega)=\left\{\begin{array}{cc}
\sqrt{p q} & \omega \in\left[0,\left(1-\frac{1}{s}\right) \frac{\pi}{p}\right) \\
\sqrt{p q} \theta\left(\frac{\omega-a}{b}\right) & \omega \in\left[\left(1-\frac{1}{s}\right) \frac{\pi}{p}, \frac{\pi}{q}\right) \\
0 & \omega \in\left[\frac{\pi}{q}, \pi\right]
\end{array}\right. \\
& G(\omega)=\left\{\begin{array}{cc}
0 & \omega \in\left[0,\left(1-\frac{1}{g}\right) \pi\right) \\
\sqrt{s} \theta_{c}\left(\frac{\omega-p a}{p b}\right) & \omega \in\left[\left(1-\frac{1}{g}\right) \pi, \frac{p}{q} \pi\right) \\
\sqrt{s} & \omega \in\left[\frac{p}{q} \pi, \pi\right]
\end{array}\right.
\end{aligned}
$$

\section{Results and Discussion}

The NIR sensor position over pancreas of diabetic person and infrared signal acquire for before meal consumption and after meal consumption. The infrared signal acquired before meal consumption is shown in figure 6.

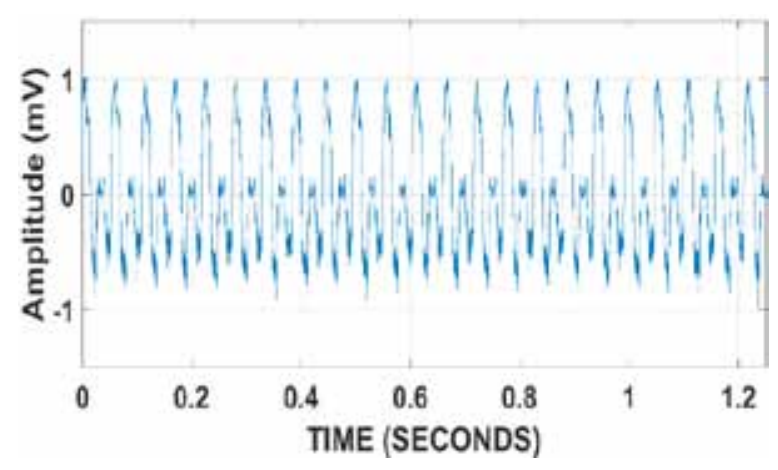

Figure 6. Pancreas NIR signal (Before meal consumption)

The acquired NIR signal decompose up to 11 levels to determine subband signal influenced by pancreas insulin secretion. The NIR signal process through RADWT for multi-resolution frequency analysis. The decomposed subband signal is shown in figure 7.Figure 8 shows the reconstructed subband signal from individual subband.

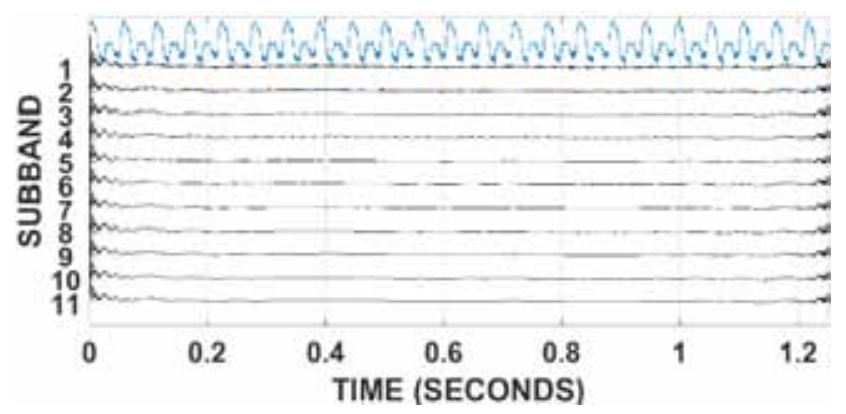

Figure 7. NIR signal decomposition with RADWT (Before meal consumption)

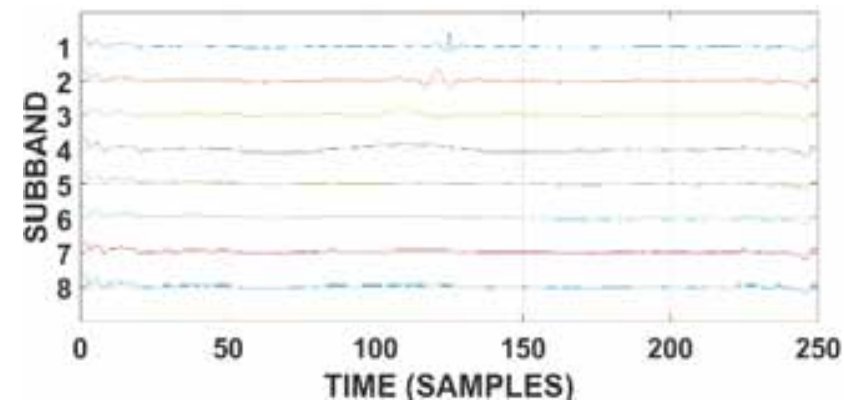

Figure 8. Reconstructed subband signal of the pancreas NIR signal (Before Meal consumption)

The multiresolution analysis evaluate for NIR signal subband energy. The RADWT, decomposes the signal and subband energy evaluate for each subband signal. The subband energy level of the NIR signal before meal consumption is shown in figure 9 . 


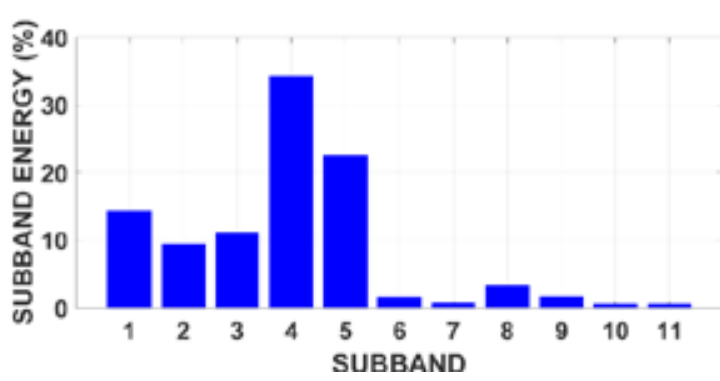

Figure 9. Subband Energy Level of the Pancreas NIR signal (Before meal consumption)

In figure 9 the subband energy level is low due to low insulin secretion from pancreas. The low insulin secretion from pancreas cause high blood glucose level. The subband energy level attains a maximum of $24 \%$ at fifth subband.

The pancreas NIR signal was obtained from pancreas with NIR sensor after mealconsumption is shown in figure 10 .

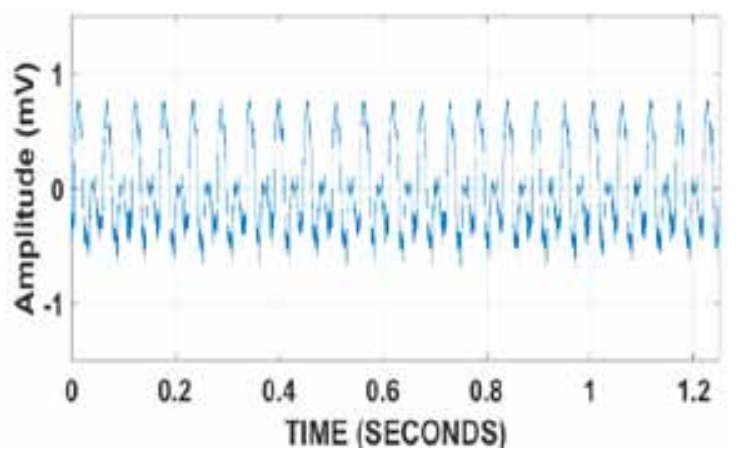

Figure 10. Pancreas NIR signal (After meal consumption)

The decomposed signal with RADWTis shown in figure 11. The subband signal reconstructed with RADWT from individual subband is shown in figure 12. In figure 12, the subband energy increases when the insulin secretion level from pancreas increase.

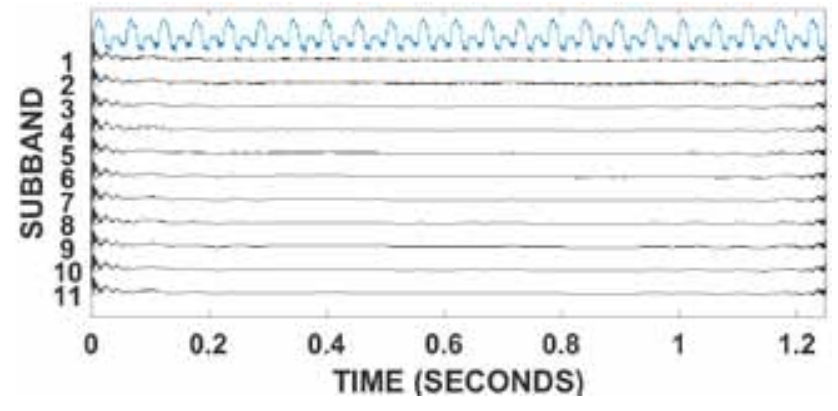

Figure 11. NIR signal decomposition with RADWT (Aftermealconsumption)

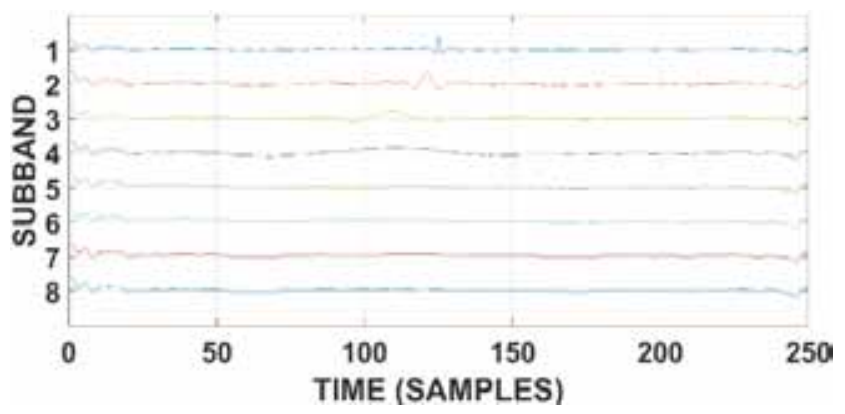

Figure 12. Reconstructed subband signal of the pancreas NIR signal (After meal consumption)

The subband energy level reconstructed NIR signal with RADWT from pancreas after meal consumption is shown in figure 13.

In figure 13, the subband energy level increases due to insulin secretion from pancreas. The subband energy level attains a maximum of $28 \%$ for fifth subband. The increase in pancreas insulin secretion lowers blood glucose level.

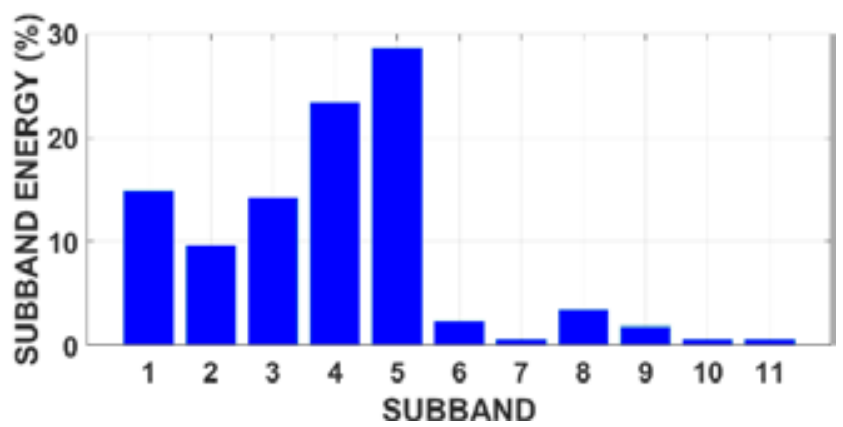

Figure 13. Subband Energy Level of the Pancreas NIR signal (After meal consumption)

The NIR signal from pancreas of non-diabetic person was obtained for before food consumption and after food consumption scenarios. The NIR subband energy variation was similar to diabetic person. However, the subband energy increased to maximum of $35 \%$ at fifth subband before food consumption and increased further to $42 \%$ after food consumptiondue to high insulin response. The subband energy increased proportional to insulin response from pancreas. The high insulin response from pancreas regulated blood sugar level. The table 1 gives information about subband energy, insulin response and glucometer reading of diabetic and nondiabetic person. 
Table 1: NIR signal parameter of diabetic and non-diabetic person.

\begin{tabular}{|l|c|c|c|c|}
\hline \multirow{2}{*}{ Parameters } & \multicolumn{2}{|c|}{ Diabetic person } & \multicolumn{2}{c|}{ Nondiabetic person } \\
\cline { 2 - 5 } & $\begin{array}{c}\text { Before meal } \\
\text { consumption }\end{array}$ & $\begin{array}{c}\text { After meal } \\
\text { consumption }\end{array}$ & $\begin{array}{c}\text { Before meal } \\
\text { consumption }\end{array}$ & $\begin{array}{c}\text { After meal } \\
\text { consumption }\end{array}$ \\
\hline Subband energy & 24 & 28 & 15 & 21 \\
\hline Glucometer values & 183 & 240 & 95 & 132 \\
\hline Lab results & 165 & 228 & 90 & 120 \\
\hline
\end{tabular}

Regression Modeling: The regression modeling is a statistical procedure used to estimate the linear or straight-line relationship that relates two or more variables. The linear relationship represents amount of change in one variable that relates with change in another variable. The regression modeling also evaluate for statistical significance, to check whether the linear relationship emerge by chance or not. The two variable regression model includes two variables such as an independent variable and a dependent variable. The independent variable causes changes in the dependent variable.

Linear Regression: Linear regression model determines relationship between two variables through fitting a linear equation forobserved data. One variable is considered as an explanatory variable, and the other is considered as a dependent variable.

The equation of a linear regression line is of the form

$$
Y=a+b X
$$

where,

$X$ is the explanatory variable.

$Y$ is the dependent variable.

The slope of line is $b$, and the intercept is $a$ (the value of $y$ when $x=0$ ). The formula for computing intercept $a$ and slope $b$ is given as

$$
\begin{aligned}
a & =\frac{\left(\sum y\right)\left(\sum x^{2}\right)-\left(\sum x\right)\left(\sum x y\right)}{n\left(\sum x^{2}\right)-\left(\sum x\right)^{2}} \\
b & =\frac{n\left(\sum x y\right)-\left(\sum x\right)\left(\sum y\right)}{n\left(\sum x^{2}\right)-\left(\sum x\right)^{2}}
\end{aligned}
$$

The blood glucose level of diabetic person obtain from subband energy and labvalues represent by equation

$$
\mathrm{Y}=14.20 * \mathrm{X}-159.0
$$

and the blood sugar level of non-diabetic person represent by

$$
\mathrm{Y}=6.393 * \mathrm{X}-1.357
$$

\section{Conclusion}

A non-invasive technique for monitoring blood glucose concentration is presented. The blood glucose level determine with insulin secretion level from pancreas, NIR sensor measures the insulation secretion level in pancreas. The intensity of the NIR light varies according to the insulin secretion level in pancreas. The NIR signal obtained from the pancreas is processed through RADWT to evaluate the insulin level in pancreas. The RADWT subband energy level is relative to insulin secretion from pancreas. The subband energy is low when the insulin secretion level in pancreas is low,before food consumption and the sub band energy level is high when insulin secretion level in pancreas increase after food consumption. The subband energy of NIR signal validates with glucometer measurement results.

Conflict of Interest: The authors declare no conflict of interest.

\section{Source of Funding: Self}

Ethical Clearance: All procedures were in accordance with the 1964 Helsinki Declaration (and its amendments). No approval by ethical committee or institutional review board was required. Informed.

\section{References}

1. Yujiro Tanaka, Takuro Tajima, Michiko Seyama, Kayo Waki. Differential Continuous Wave Photoacoustic Spectroscopy for Non-Invasive Glucose Monitoring. IEEE Sensors Journal 2020;20(8): 4453-4458. 
2. Sandeep Kumar Chamoli, Subhash Chandra Singh, Chunlei Guo. Design of Extremely Sensitive Refractive Index Sensors in Infrared for Blood Glucose Detection. IEEE Sensors Journal 2020; 20(9): 4628-4634.

3. Abhishek Kandwal, Zedong Nie, Tobore Igbe, Jingzhen Li, Yuhang Liu, Louis WY Liu, Yang Hao. Surface Plasmonic Feature Microwave Sensor with Highly Confined fields for AqueousGlucose and Blood-Glucose Measurements. IEEE Transactions on Instrumentation and Measurement 2020; 9456(c): 1-9.

4. Jingzhen Li, Tobore Igbe, Yuhang Liu, Zedong Nie, Wenjian Qin, Lei Wang, Yang Hao. An Approach for Noninvasive Blood Glucose Monitoring Based on Bioimpedance Difference Considering Blood Volume Pulsation. IEEE Access 2018; 6: 5111951129.

5. Maria Celeste Cebedio, Lucas Andres Rabioglio, Ivan Exequiel Gelosi, Ramiro Avalos Ribas, Alejandro Jose Uriz, Jorge Castineira Moreira. Analysis and Design of a Microwave Coplanar Sensor for Non-Invasive Blood Glucose Measurements. IEEE Sensors Journal 2020; 20(18): 10572-10581.

6. Ala Eldin Omer, George Shaker, Safieddin SafaviNaeini, Kevin Murray, Richard Hughson. Glucose Levels Detection Using mm-Wave Radar. IEEE Sensors Letters 2017; 2(3): 1-4.

7. Shyqyri Haxha, Jaspreet Jhoja. Optical Based Noninvasive Glucose Monitoring Sensor Prototype. IEEE Photonics Journal 2016; 8(6):1-11.

8. Shao Ying Huang, Omkar, Yu Yoshida, Adan Garcia, Xavier Chia, Wen Chuan Mu, Yu Song Meng, Wenwei Yu. Microstrip Line-Based Glucose Sensor for Noninvasive Continuous Monitoring using the Main Field for Sensing and Multivariable Crosschecking. IEEE Sensors Journal 2018; 19(2): 535-547.
9. Joseph Y Lucisano, Timothy L Routh, Joe T Lin, David A Gough. Glucose Monitoring in Individuals with Diabetes Using a Long-Term Implanted Sensor/Telemetry System and Model. IEEE Trans Biomed Eng 2017; 64(9): 1982-1993.

10. Ala Eldin Omer, George Shaker, Safieddin SafaviNaeini. Portable Radar-Driven Microwave Sensor for Intermittent Glucose Levels Monitoring. IEEE Sensors Letters 2020; 4(5): 21-24.

11. Sina Kiani, Pejman Rezaei, Mahmood Karami,R. A. Sadeghzadeh. Band-stop filter sensor based on SIW cavity for the non-invasive measuring of blood glucose. IET wireless Sensor Systems 2019; 9(1): $1-5$.

12. Hara Prasada Tripathy, Priyabrata Pattanaik, Sushanta Kumar Kamilla, Rajesh Kumar Tripathy. A Simulation Approach to Study the Effect of Ultrasonic MEMS Based Receiver for Blood Glucose Sensing Applications. IEEE Sensors Letters 2017; 1(5): 1-4.

13. Volkan Turgul, Izzet Kale. Simulating the Effects of Skin Thickness and Fingerprints to Highlight Problems with Non-Invasive RF Blood Glucose Sensing from Fingertips. IEEE Sensors Journal 2017; 17(22): 7553-7560.

14. Gang Wang, Michael D Poscente, Simon S Park, Christopher N Andrews, Orly Yadid-Pecht, Martin P Mintchev. Wearable Microsystem for Minimally Invasive, Pseudo-Continuous Blood Glucose Monitoring: The e-Mosquito. IEEE Transactions on Biomedical Circuits and Systems 2017; 11(5): 979-987.

15. Zhibin Xiao, Xi Tan, Xianliang Chen, Sizheng Chen, Zijian Zhang, Hualei Zhang, Junyu Wng, Yue Huang, Peng Zhang, Lirong Zheng, Hao Min. An implantable RFID sensor tag toward continuous glucose monitoring. IEEE Journal of Biomedical and Health Informatics 2015; 19(3): 910-919. 